\title{
Anisotropic quasiparticle scattering rates in slightly underdoped to optimally doped high-temperature $\mathrm{La}_{2-x} \mathrm{Sr}_{x} \mathrm{CuO}_{4}$ superconductors
}

\author{
J. Chang, ${ }^{1}$ M. Shi, ${ }^{2}$ S. Pailhés, ${ }^{1}$ M. Månsson, ${ }^{3}$ T. Claesson, ${ }^{3}$ O. Tjernberg, ${ }^{3}$ A. Bendounan, ${ }^{1}$ Y. Sassa, ${ }^{1}$ L. Patthey, ${ }^{2}$ \\ N. Momono, ${ }^{4}$ M. Oda,${ }^{4}$ M. Ido, ${ }^{4}$ S. Guerrero, ${ }^{5}$ C. Mudry, ${ }^{5}$ and J. Mesot ${ }^{1, *}$ \\ ${ }^{1}$ Laboratory for Neutron Scattering, ETH Zurich and Paul Scherrer Institute, CH-5232 Villigen PSI, Switzerland \\ ${ }^{2}$ Swiss Light Source, Paul Scherrer Institute, CH-5232 Villigen PSI, Switzerland \\ ${ }^{3}$ Materials Physics, Royal Institute of Technology KTH, S-164 40 Kista, Sweden \\ ${ }^{4}$ Department of Physics, Hokkaido University, Sapporo 060-0810, Japan \\ ${ }^{5}$ Condensed Matter Theory Group, Paul Scherrer Institute, CH-5232 Villigen PSI, Switzerland
}

(Received 9 October 2008; published 4 November 2008)

\begin{abstract}
An angle-resolved photoemission study of the scattering rate in the superconducting phase of the hightemperature superconductor $\mathrm{La}_{2-x} \mathrm{Sr}_{x} \mathrm{CuO}_{4}$ with $x=0.145$ and $x=0.17$, as a function of binding energy and momentum, is presented. We observe that the scattering rate scales linearly with binding energy up to the high-energy scale $E_{1} \sim 0.4 \mathrm{eV}$. The scattering rate is found to be strongly anisotropic, with a minimum along the $(0,0)-(\pi, \pi)$ direction. A possible connection to a quantum-critical point is discussed.
\end{abstract}

DOI: 10.1103/PhysRevB.78.205103

PACS number(s): 74.72.Dn, 74.25.Jb, 79.60.-i

\section{INTRODUCTION}

Angle-resolved photoelectron spectroscopy (ARPES) is a powerful probe of electronic interactions in solids. For example, in studies of high-temperature superconductors (HTSCs), a low-energy kink, herein denoted $E_{0}$, was observed in the $(0,0)$ to $(\pi, \pi)$ direction (nodal direction) of the quasiparticle (QP) spectra. ${ }^{1,2}$ Recently, the high-energy part $(0.2-1.5 \mathrm{eV})$ of the ARPES spectra has attracted considerable attention. ${ }^{3-9}$ Graf et al. ${ }^{3}$ reported the existence of two high-energy anomalies $E_{1}$ and $E_{2}$ in the nodal dispersion. These three anomalies in the nodal spectra, $E_{0} \approx 0.06 \mathrm{eV}$, $E_{1} \approx 0.4 \mathrm{eV}$, and $E_{2} \approx 0.8 \mathrm{eV}$, seem to be universal for the cuprates and they have been interpreted in terms of manybody interactions. ${ }^{10-15}$ We showed in Ref. 6 that the highenergy anomaly $E_{1}$ exists throughout the whole Brillouin zone (BZ) and that $E_{1}$ disperses continuously as one moves from the nodal to the antinodal $[(0,0)-(\pi, 0)]$ direction.

Transport measurements have revealed anomalous normal-state (NS) properties of optimally doped HTSCs. The NS resistivity, at optimal doping, is found to scale linearly with the temperature $T$ up to $T \sim 1000 \mathrm{~K} .{ }^{16}$ This part of the phase diagram is therefore also known as the strange-metal phase. Although anomalous NS properties have been described successfully by the marginal Fermi-liquid (MFL) phenomenology, ${ }^{17}$ there is still no consensus for the underlying interactions responsible for HTSCs and these anomalous properties.

In this paper, we investigate the $\mathrm{QP}$ scattering rate in $\mathrm{La}_{2-x} \mathrm{Sr}_{x} \mathrm{CuO}_{4}$ (LSCO) close to optimally doping. Our main findings, valid for energies much larger than the superconducting gap, are twofold. First, the dominant scattering channel scales linearly with the binding energy $\omega$. Second, this scattering channel is highly anisotropic, exhibiting a sharp minimum along the nodal direction. We emphasize that while these results do not elucidate the pairing mechanism of HTSCs, they provide constraints to any theory on the strange-metal phase.

\section{METHODS}

Single crystals of LSCO with $x=0.145$ and $x=0.17$ were grown using the traveling-solvent floating-zone method. ${ }^{18}$ Both samples have a transition temperature $T_{c} \approx 36 \mathrm{~K}$ with $\Delta T_{c} \approx 1.5 \mathrm{~K}$. The ARPES experiments were performed on the Surface/Interface Spectroscopy (SIS) beamline at the Swiss Light Source of the Paul Scherrer Institute. The spectra were acquired with a Scienta SES2002 electron analyzer, which was calibrated by recording spectra from polycrystalline copper on the sample holder. The measurements were performed at $T=15 \mathrm{~K}$ under ultrahigh vacuum using $55 \mathrm{eV}$ circularly polarized photons with an energy resolution of 17-40 meV. Data were recorded in the second BZ but are presented in the first $\mathrm{BZ}$, for convenience.

\section{RESULTS}

Starting with the low-energy properties, we plot in Figs. 1(a) and 1(b) the ARPES intensity as a function of binding energy and electron momentum $\mathbf{k}$, along cuts through the nodal and antinodal points, respectively. The nodal spectrum is characterized by sharp peaks and the leading edge of the energy distribution curve (EDC) at $\mathbf{k}_{F}$ reaches the Fermi level $E_{F}$. The momentum distribution curve (MDC) linewidths are much broader in the antinodal spectrum, and the leading edge of the EDCs at $\mathbf{k}_{F}$ is shifted away from $E_{F}$ due to the presence of an energy gap $\Delta .{ }^{19}$ The double peak structure of antinodal MDC linewidth stems from the cut crossing two branches of the QP dispersion.

In Fig. 2(a) we show the ARPES intensity up to very high binding energy for the nodal cut shown in the inset. The background was subtracted and the intensity was normalized to the maximum intensity of the MDCs for each energy step. The open black squares indicate the dispersion extracted from MDC analysis according to Fig. 2(b) (the blue lines will be explained below). As previously reported, ${ }^{3}$ the nodal spectrum exhibits two high-energy anomalies, $E_{1}$ and $E_{2}$, as indicated by black arrows in Fig. 2(a). For $E_{1}<\omega<E_{2}$ the 


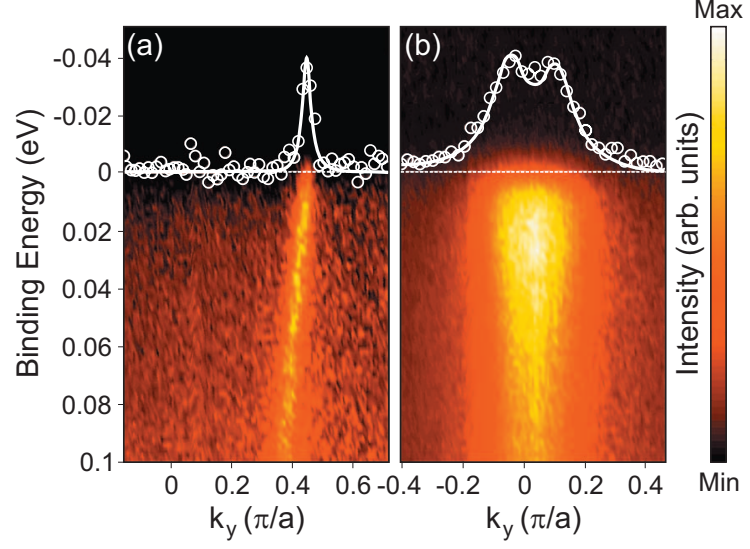

FIG. 1. (Color) [(a) and (b)] ARPES intensity, recorded on $x$ $=0.145$, for nodal and antinodal cuts, respectively. The white points are the MDC at $E_{F}$. The intensity ratio between nodal and antinodal is $\sim 1 / 3$.

MDC peaks are pinned at $\mathbf{k}_{\mathrm{WF}} \approx(1 / 4,1 / 4)$, while for $\omega$ $>E_{2}$ the MDC peaks disperse again. These anomalies have become known as the waterfall (WF) feature. Herein, the waterfall refers only to the $E_{1}\left(\phi_{\mathrm{WF}}\right)$ anomaly, and we use the notation $\mathbf{k}_{\mathrm{WF}}=(|\mathbf{k}|, \phi)$, with the polar angle $\phi$ defined from the $Y$ point as shown in Fig. 3(c). This paper is dedicated to the study of the QP scattering rate $\operatorname{Im} \Sigma(\phi, \omega)$ that we model by assuming that it is the product of the MDC linewidth $\Gamma(\phi, \omega)$ and a characteristic velocity $v(\phi)$, to be defined more precisely below. The polar angle $\phi$ here, to a first approximation, labels the cut along which the linewidth is measured. We limit our analysis to $\omega<0.6 \mathrm{eV}$, where welldefined Lorentzian-shaped peaks, on nearly flat background, are observed in the MDC, as shown in Fig. 2(b). We examine the low- and high-energy dependence of the half-width at
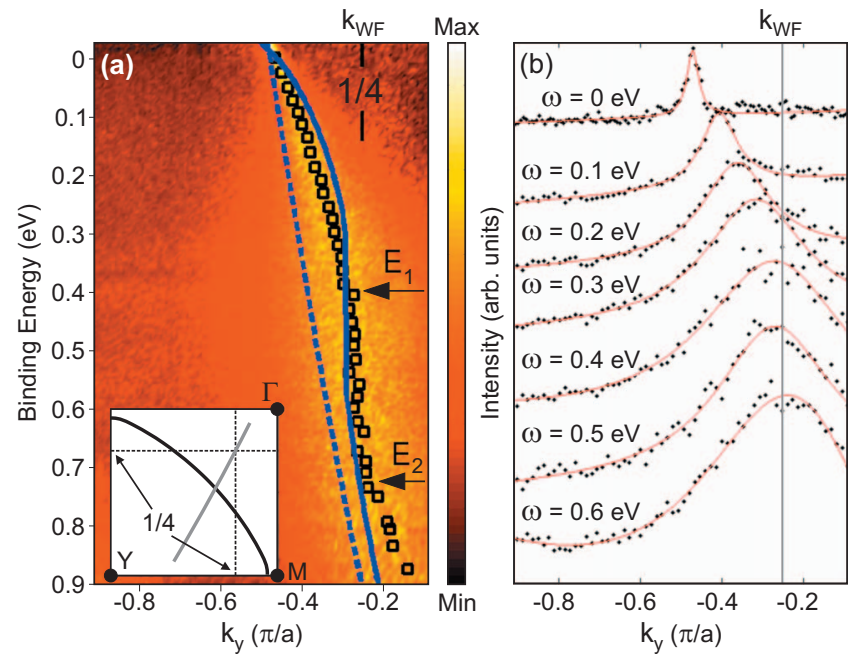

FIG. 2. (Color) (a) MDC-normalized ARPES spectra, recorded on $x=0.145$, for the nodal cut shown in the inset. Black squares represent the MDC peak positions. Dashed blue line represents the bare-band dispersion $\varepsilon_{\mathbf{k}}$. Solid blue line represents the renormalized dispersion obtained from discussions below. (b) MDCs for $\omega$ up to $0.6 \mathrm{eV}$. The red lines represent fits to the data with a Lorentzian line shape on a sloping background.

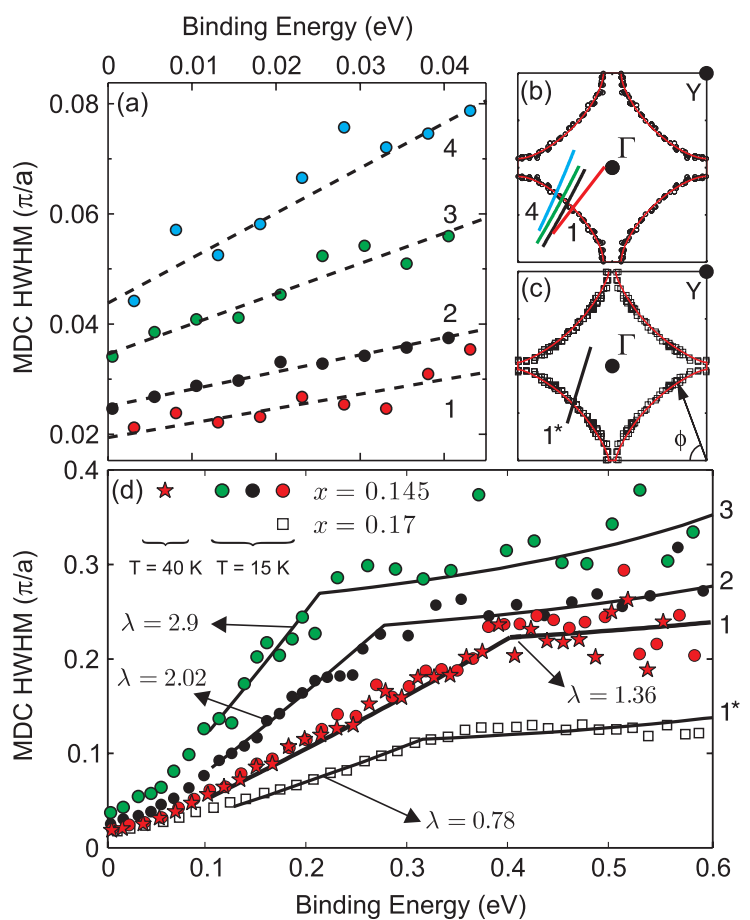

FIG. 3. (Color) (a) Low-energy dependence of the MDC linewidths for the cuts shown in (b). Dashed lines represent linear fits of the measured scattering rate. [(b) and (c)] Fermi surface of $x$ $=0.145$ and $x=0.17$ samples, respectively. The red lines are tightbinding fit to the data. (d) $\omega$ dependence of MDC linewidth for cuts 1-3 in (b) and the cut in (c). Cut 1 in (b) was measured both in the superconductor (SC) state (circular points) and in the NS (star points) at $T=40 \mathrm{~K}$. The solid lines are $\operatorname{Im} \Sigma(\omega) / v_{\phi}$, where $\operatorname{Im} \Sigma(\omega)$ and $v_{\phi}$ are given in the text.

half maximum (HWHM) $\Gamma(\phi, \omega)$ extracted from Lorentzian fits to the MDC from Figs. 3(a) and 3(d). The Fermi surfaces of LSCO with $x=0.145$ and $x=0.17$ shown in Figs. $3(\mathrm{~b})$ and 3 (c), respectively, are consistent with previous reports. ${ }^{20}$ The color code of the cuts in Figs. 3(b) and 3(c) is the same as that in Figs. 3(a) and 3(d).

Before studying $\Gamma(\phi, \omega)$, we first discuss the $\phi$ dependence of the high-energy anomaly $E_{1}$. Figure 4(a) shows $E_{1}$ extracted from the anomaly in the scattering rate shown in Fig. 3(d). $E_{1}\left(\phi_{\mathrm{WF}}\right)$ disperses strongly and we have previously suggested the following phenomenological form:

$$
E_{1}\left(\phi_{\mathrm{WF}}\right)=E_{1}(\pi / 4)\left[1-\left|\cos \left(2 \phi_{\mathrm{WF}}\right)\right|\right],
$$

with $E_{1}(\pi / 4)=0.43 \mathrm{eV} .^{6}$ Within the experimental uncertainty, there is no significant difference between $E_{1}\left(\phi_{\mathrm{WF}}\right)$ for LSCO with $x=0.145$ and $x=0.17$.

The energy scales $E_{0}$ and $E_{1}$ define three distinct characteristic regimes shown in Fig. 4(a). Regime I is the lowenergy regime $E_{F}<\omega<\min \left\{E_{0}, E_{1}\left(\phi_{\mathrm{WF}}\right)\right\}$, followed by an intermediate regime II defined as $E_{0}<\omega<E_{1}\left(\phi_{\mathrm{WF}}\right)$. Finally, we define the high-energy regime III as $E_{1}\left(\phi_{\mathrm{WF}}\right)<\omega$.

Although the main purpose of this paper is to study the QP scattering rate $\Sigma(\phi, \omega)$ in regime II, we present $\Gamma(\phi, \omega)$ in the three regimes. The MDC linewidth $\Gamma(\phi, \omega)$ in regimes I and II obeys 


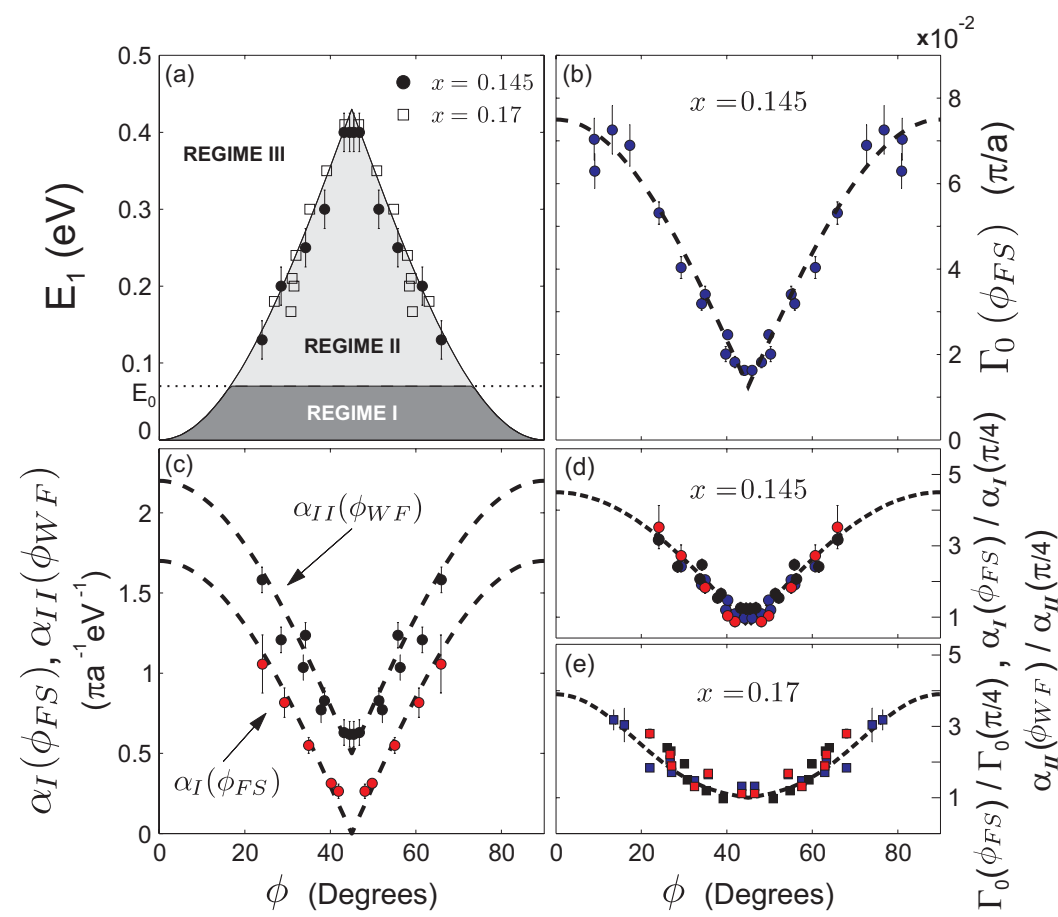

$$
\Gamma_{i}(\phi, \omega)=\Gamma_{i}^{0}(\phi)+\alpha_{i}(\phi) \omega, \quad i=\mathrm{I}, \mathrm{II} .
$$

$$
\begin{aligned}
\varepsilon_{\mathbf{k}}= & -2 t\left[\cos k_{x} a+\cos k_{y} a\right]-4 t^{\prime} \cos k_{x} a \cos k_{y} a \\
& -2 t^{\prime \prime}\left[\cos 2 k_{x} a+\cos 2 k_{y} a\right]-\mu,
\end{aligned}
$$

Consistent with previous ARPES (Ref. 21) and transport ${ }^{22}$ measurements, the elastic term $\Gamma_{\mathrm{I}}^{0}\left(\phi_{\mathrm{FS}}\right)$ is anisotropic as shown in Fig. 4(b). The parameter $\alpha_{\mathrm{I}}\left(\phi_{\mathrm{FS}}\right)$, related to the inelastic scattering, is analyzed by linear fits to the scattering rate $\Gamma_{\mathrm{I}}\left(\phi_{\mathrm{FS}}, \omega\right)$; see dashed lines in Fig. 3(a). We show in Fig. 4(c) the $\phi_{\mathrm{FS}}$ dependence of $\alpha_{\mathrm{I}}\left(\phi_{\mathrm{FS}}\right)$ in the vicinity of the nodal point. The linear dependence of $\Gamma_{\mathrm{I}}\left(\phi_{\mathrm{FS}}, \omega\right)$ was also observed in Bi2212 (Refs. 23-25) and interpreted in Ref. 25 as a signature of the $d$-wave nodes.

We now turn to regime II, for which $\Gamma_{\mathrm{II}}^{0}(\phi)$ is negligible for $x=0.145$ and the angular dependence of $\alpha_{\mathrm{II}}\left(\phi_{\mathrm{WF}}\right)$ is shown in Fig. 3(c). Observe that the coefficient $\alpha_{\mathrm{II}}$ is the same for both $T_{c}>T=15 \mathrm{~K}$ and $T_{c}<T=40 \mathrm{~K}$; see Fig. 3(d). This is expected since the relevant energy scale in regime II is an order of magnitude larger than the maximum of the superconducting gap. Hence the linear dependence on $\omega$ in Eq. (2) cannot be attributed to the $d$-wave nodes. Nevertheless and remarkably $\Gamma_{\mathrm{I}}^{0}, \alpha_{\mathrm{I}}$, and $\alpha_{\mathrm{II}}$ follow a very similar angular dependence. To show this, we plot $\Gamma_{0}\left(\phi_{\mathrm{FS}}\right) / \Gamma_{0}(\pi / 4)$, $\alpha_{I}\left(\phi_{\mathrm{FS}}\right) / \alpha_{\mathrm{I}}(\pi / 4)$, and $\alpha_{\mathrm{II}}\left(\phi_{\mathrm{WF}}\right) / \alpha_{\mathrm{II}}(\pi / 4)$ in Fig. $4(\mathrm{~d})$. For $\mathrm{La}_{1.83} \mathrm{Sr}_{0.17} \mathrm{CuO}_{4}$ we find the same anisotropic dependence, although with a slightly weaker and flatter dependence around the nodal direction; see Fig. 4(e).

The approximate Lorentzian shape of the MDCs suggests that one can neglect the $\mathbf{k}$ and $\omega$ dependence of the photoelectron matrix elements. If so, we can approximate the ARPES intensity by the single-particle spectral function

$$
A(\mathbf{k}, \omega)=\frac{1}{\pi} \frac{-\operatorname{Im} \Sigma(\mathbf{k}, \omega)}{\left[\omega-\operatorname{Re} \Sigma(\mathbf{k}, \omega)-\varepsilon_{\mathbf{k}}\right]^{2}+[\operatorname{Im} \Sigma(\mathbf{k}, \omega)]^{2}} .
$$

Here $\Sigma(\mathbf{k}, \omega)$ is the self-energy and $\varepsilon_{\mathbf{k}}$ is the bare-band dispersion. We model $\varepsilon_{\mathbf{k}}$ with the tight-binding dispersion
FIG. 4. (Color) (a) Momentum dependence of . Solid line is obtained from Eq. (1). (b) MDC ( $\alpha_{\mathrm{ISS}}$. (c) Angular dependence of $\alpha_{\mathrm{I}}(\phi)$ $\alpha_{\mathrm{I}}\left(\phi_{\mathrm{FS}}\right) / \alpha_{\mathrm{I}}(\pi / 4)$, and $\alpha_{\mathrm{II}}\left(\phi_{\mathrm{WF}}\right) / \alpha_{\mathrm{II}}(\pi / 4)$ for $x$ $=0.145$ and $x=0.17$, respectively. Blue, red, and black points are $\Gamma_{0}, \alpha_{\mathrm{I}}$, and $\alpha_{\mathrm{II}}$, respectively. Data have been symmetrized with respect to the nodal direction. Dashed lines are guides for the eyes. where $\mu$ is the chemical potential and $t, t^{\prime}$, and $t^{\prime \prime}$ denote nearest-, second-nearest-, and third-nearest-neighbor hopping integrals on a square lattice, respectively. The ratios $\mu / t, t^{\prime} / t$, and $t^{\prime \prime} / t$, given in Table $\mathrm{I}$, are chosen such that $\varepsilon_{\mathbf{k}}=0$ fits the experimentally determined Fermi surfaces; see Figs. 3(b) and 3(c). Assuming that the bandwidth $t$ varies slowly within the doping range of interest, we use for the bare band $\varepsilon_{\mathbf{k}}$ (see dashed blue line in Fig. 2) $t=0.48 \mathrm{eV}$ for both $x=0.145$ and $x=0.17 .^{26}$

In regime II we analyze the cuts shown in Figs. 3(b) and 3(c) with a generalized MFL self-energy,

$$
\operatorname{Im} \Sigma\left(\phi_{\mathrm{WF}}, \omega\right)=\frac{-\lambda\left(\phi_{\mathrm{WF}}\right) \pi}{2} \begin{cases}|\omega|, & |\omega|<\omega_{c}\left(\phi_{\mathrm{WF}}\right) \\ \omega_{c}\left(\phi_{\mathrm{WF}}\right), & |\omega|>\omega_{c}\left(\phi_{\mathrm{WF}}\right),\end{cases}
$$

and

$$
\operatorname{Re} \Sigma\left(\phi_{\mathrm{WF}}, \omega\right)=-\lambda\left(\phi_{\mathrm{WF}}\right)\left[\omega \ln \left(\frac{\omega_{c}\left(\phi_{\mathrm{WF}}\right)}{\omega}\right)+\cdots\right] .
$$

The conventional MFL ansatz ${ }^{10,17}$ for the self-energy assumes that the dimensionless coupling $\lambda$ and the characteristic energy cutoff $\omega_{c}$ are $\phi$ independent. Motivated by Eq. (2), we are going to relax this assumption in order to de-

TABLE I. Tight-binding parameters for $\mathrm{La}_{2-x} \mathrm{Sr}_{x} \mathrm{CuO}_{4}$.

\begin{tabular}{lccccc}
\hline \hline Compound & $t(\mathrm{eV})$ & $\mu / t$ & $t^{\prime} / t$ & $t^{\prime \prime} / t$ & $E_{1}(\pi / 4) / t$ \\
\hline$x=0.145$ & 0.48 & 0.68 & -0.125 & 0.078 & 0.9 \\
$x=0.17$ & 0.48 & 0.84 & -0.144 & 0.072 & 0.9 \\
\hline \hline
\end{tabular}


scribe the MDC linewidth in Fig. 3 from Eqs. (3)-(6). Along the cuts shown in Figs. 3(b) and 3(c), the MDCs have a Lorentzian shape with $\operatorname{HWHM} \Gamma(\phi, \omega)=\operatorname{Im} \Sigma(\phi, \omega) / v_{\phi}$, where $v_{\phi}=d \varepsilon_{\mathbf{k}} / d \mathbf{k}$ is the velocity along the cut. ${ }^{27}$ Combining Eqs. (2) and (5), it then follows that

$$
\pi \lambda\left(\phi_{\mathrm{WF}}\right)=\alpha_{\mathrm{II}}\left(\phi_{\mathrm{WF}}\right) v_{\phi} \approx \alpha_{\mathrm{II}}\left(\phi_{\mathrm{WF}}\right) v_{\phi_{\mathrm{WF}}} .
$$

This approximation is valid in the vicinity of the nodal point where the bare-band velocity $v_{\phi}$ is weakly dependent on $\mathbf{k}$ for $\omega<0.6 \mathrm{eV}$ but breaks down upon approaching the Van Hove singularity of $\varepsilon_{\mathbf{k}}$ in the antinodal region. Second, we approximate the cutoff energy by

$$
\omega_{c}\left(\phi_{\mathrm{WF}}\right) \approx E_{1}\left(\phi_{\mathrm{WF}}\right) .
$$

Now, the renormalized dispersion is the solution of $\omega_{p}(\mathbf{k})$ $=\operatorname{Re} \Sigma\left[\omega_{p}(\mathbf{k})\right]+\varepsilon_{\mathbf{k}}$. In this fashion we obtain a consistent agreement for both the renormalized dispersion [solid blue line in Fig. 2(a)] and the MDC linewidth [solid lines in Fig. $3(\mathrm{~d})$ ]. Thus, in contrast to earlier claims, ${ }^{28}$ we have shown that the WF features can be described by a Kramers-Kronig consistent self-energy function $\Sigma(\phi, \omega)$. We would like to stress that the $\alpha_{\mathrm{II}}\left(\phi_{\mathrm{WF}}\right)$ and $v_{\phi_{\mathrm{WF}}}$ dependencies on $\phi_{\mathrm{WF}}$ do not cancel out, leaving a net anisotropic coupling parameter $\lambda\left(\phi_{\mathrm{WF}}\right)$. Furthermore, the observation that $\alpha_{\mathrm{II}}(\phi)$ has a stronger dependence on doping than $\varepsilon_{\mathbf{k}}$ implies that the coupling constant $\lambda\left(\phi_{\mathrm{WF}}\right)$ decreases with overdoping.

\section{DISCUSSION}

We have shown that both the elastic- and inelasticscattering rates are highly anisotropic. However, an isotropic channel may be hidden by the dominant anisotropic scattering channel. Recently, two scattering channels have been identified, in the overdoped regime of $\mathrm{Tl}_{2} \mathrm{Ba}_{2} \mathrm{CuO}_{\delta+6}$ (T12201), by an angular magnetoresistance oscillation (AMRO) study. ${ }^{29}$ One channel, related to electron-electron scattering, is isotropic and exhibits $T^{2}$ dependence. A second channel, of unknown origin, is anisotropic and depends linearly on $T$. For even more overdoped samples, resistivity measurements on $\mathrm{La}_{2-x} \mathrm{Sr}_{x} \mathrm{CuO}_{4}$ have demonstrated that the electron-electron scattering channel is completely dominant. ${ }^{29,30}$

The picture that emerges from this work and previous transport measurements ${ }^{29,30}$ is the following. In the underdoped regime the dominant scattering channel is highly anisotropic and exhibits MFL behavior. Upon further hole doping this channel gradually decreases and conventional electron-electron interactions become increasingly important. Eventually in the extremely overdoped regime, $x>0.3$, electron-electron interactions are the dominant scattering mechanism.

\section{CONCLUSION}

In conclusion, we have presented a comprehensive study of the $\omega$ and $\mathbf{k}$ dependence of the scattering rate in the vicinity of optimally doped $\mathrm{La}_{2-x} \mathrm{Sr}_{x} \mathrm{CuO}_{4}$. The dominant inelastic-scattering channel scales linearly with $\omega$ up to the onset of the waterfall feature. Remarkably, both the elasticand inelastic-scattering channels are strongly anisotropic, with minima along the nodal direction. This anisotropic MFL behavior can be used to discriminate between competing theories for the strange-metallic phase in high-temperature superconductors that rely on the single-band Hubbard model, ${ }^{31}$ the existence of a quantum-critical point, ${ }^{32-35}$ or the separation of spin and charge quantum numbers. ${ }^{36,37}$

\section{ACKNOWLEDGMENTS}

This work was supported by the Swiss National Science Foundation (through NCCR, MaNEP, and Grants No. 200020-105151 and No. PBEZP2-122855), the Ministry of Education and Science of Japan, and the Swedish Research Council. This work was performed at SLS of the Paul Scherrer Institute, Villigen PSI, Switzerland. We thank the beamline staff of X09LA for their excellent support, and we are grateful to Lijun Zhu and Chandra Varma for enlightening discussions.
*Also at Institut de la materière complexe, Ecole Polytechnique Fédérale de Lausanne (EPFL), CH-1015 Lausanne, Switzerland. joel.mesot@psi.ch

${ }^{1}$ A. Kaminski, J. Mesot, H. Fretwell, J. C. Campuzano, M. R. Norman, M. Randeria, H. Ding, T. Sato, T. Takahashi, T. Mochiku, K. Kadowaki, and H. Hoechst, Phys. Rev. Lett. 84, 1788 (2000).

${ }^{2}$ A. Lanzara, P. V. Bogdanov, X. J. Zhou, S. A. Kellar, D. L. Feng, E. D. Lu, T. Yoshida, H. Eisaki, A. Fujimori, K. Kishio, J.-I. Shimoyama, T. Noda, S. Uchida, Z. Hussain, and Z.-X. Shen, Nature (London) 412, 510 (2001).

${ }^{3}$ J. Graf, G.-H. Gweon, K. McElroy, S. Y. Zhou, C. Jozwiak, E. Rotenberg, A. Bill, T. Sasagawa, H. Eisaki, S. Uchida, H. Takagi, D.-H. Lee, and A. Lanzara, Phys. Rev. Lett. 98, 067004 (2007).
${ }^{4}$ F. Ronning, K. M. Shen, N. P. Armitage, A. Damascelli, D. H Lu, Z.-X. Shen, L. L. Miller, and C. Kim, Phys. Rev. B 71, 094518 (2005).

${ }^{5}$ B. P. Xie, K. Yang, D. W. Shen, J. F. Zhao, H. W. Ou, J. Wei, S. Y. Gu, M. Arita, S. Qiao, H. Namatame, M. Taniguchi, N. Kaneko, H. Eisaki, K. D. Tsuei, C. M. Cheng, I. Vobornik, J. Fujii, G. Rossi, Z. Q. Yang, and D. L. Feng, Phys. Rev. Lett. 98, 147001 (2007).

${ }^{6}$ J. Chang, S. Pailhés, M. Shi, M. Månsson, T. Claesson, O. Tjernberg, J. Voigt, V. Perez, L. Patthey, N. Momono, M. Oda, M. Ido, A. Schnyder, C. Mudry, and J. Mesot, Phys. Rev. B 75, 224508 (2007).

${ }^{7}$ Z.-H. Pan, P. Richard, A. V. Fedorov, T. Kondo, T. Takeuchi, S. L. Li, Pengcheng Dai, G. D. Gu, W. Ku, Z. Wang, and H. Ding, arXiv:cond-mat/0610442 (unpublished). 
${ }^{8}$ T. Valla, T. E. Kidd, W.-G. Yin, G. D. Gu, P. D. Johnson, Z.-H. Pan, and A. V. Fedorov, Phys. Rev. Lett. 98, 167003 (2007).

${ }^{9}$ W. Meevasana, X. J. Zhou, S. Sahrakorpi, W. S. Lee, W. L. Yang, K. Tanaka, N. Mannella, T. Yoshida, D. H. Lu, Y. L. Chen, R. H. He, Hsin Lin, S. Komiya, Y. Ando, F. Zhou, W. X. Ti, J. W. Xiong, Z. X. Zhao, T. Sasagawa, T. Kakeshita, K. Fujita, S. Uchida, H. Eisaki, A. Fujimori, Z. Hussain, R. S. Markiewicz, A. Bansil, N. Nagaosa, J. Zaanen, T. P. Devereaux, and Z.-X. Shen, Phys. Rev. B 75, 174506 (2007).

${ }^{10}$ Lijun Zhu, Vivek Aji, Arkady Shekhter, and C. M. Varma, Phys. Rev. Lett. 100, 057001 (2008).

${ }^{11}$ Efstratios Manousakis, Phys. Rev. B 75, 035106 (2007).

${ }^{12}$ F. Tan, Y. Wan, and Q.-H. Wang, Phys. Rev. B 76, 054505 (2007).

${ }^{13}$ Alexandru Macridin, M. Jarrell, Thomas Maier, and D. J. Scalapino, Phys. Rev. Lett. 99, 237001 (2007).

${ }^{14}$ R. S. Markiewicz, S. Sahrakorpi, and A. Bansil, Phys. Rev. B 76, 174514 (2007).

${ }^{15}$ K. Byczuk, M. Kollar, K. Held, Y. F. Yang, I. A. Nekrasov, T. Pruschke, and D. Vollhardt, Nat. Phys. 3, 168 (2007).

${ }^{16}$ H. Takagi, B. Batlogg, H. L. Kao, J. Kwo, R. J. Cava, J. J. Krajewski, and W. F. Peck, Jr., Phys. Rev. Lett. 69, 2975 (1992).

${ }^{17}$ C. M. Varma, P. B. Littlewood, S. Schmitt-Rink, E. Abrahams, and A. E. Ruckenstein, Phys. Rev. Lett. 63, 1996 (1989).

${ }^{18}$ T. Nakano, N. Momono, M. Oda, and M. Ido, J. Phys. Soc. Jpn. 67, 2622 (1998).

${ }^{19}$ M. Shi, J. Chang, S. Pailhés, M. R. Norman, J. C. Campuzano, M. Månsson, T. Claesson, O. Tjernberg, A. Bendounan, L. Patthey, N. Momono, M. Oda, M. Ido, C. Mudry, and J. Mesot, Phys. Rev. Lett. 101, 047002 (2008).

${ }^{20}$ T. Yoshida et al., Phys. Rev. B 74, 224510 (2006).

${ }^{21}$ T. Yoshida et al., J. Phys.: Condens. Matter 19, 125209 (2007).

${ }^{22}$ A. Narduzzo, G. Albert, M. M. J. French, N. Mangkorntong, M. Nohara, H. Takagi, and N. E. Hussey, Phys. Rev. B 77, 220502(R) (2008).

${ }^{23}$ A. Kaminski, H. M. Fretwell, M. R. Norman, M. Randeria, S.
Rosenkranz, U. Chatterjee, J. C. Campuzano, J. Mesot, T. Sato, T. Takahashi, T. Terashima, M. Takano, K. Kadowaki, Z. Z. Li, and H. Raffy, Phys. Rev. B 71, 014517 (2005).

${ }^{24}$ T. Valla, T. E. Kidd, J. D. Rameau, H.-J. Noh, G. D. Gu, P. D. Johnson, H.-B. Yang, and H. Ding, Phys. Rev. B 73, 184518 (2006).

${ }^{25}$ T. Yamasaki, K. Yamazaki, A. Ino, M. Arita, H. Namatame, M. Taniguchi, A. Fujimori, Z.-X. Shen, M. Ishikado, and S. Uchida, Phys. Rev. B 75, 140513(R) (2007).

${ }^{26}$ E. Pavarini, I. Dasgupta, T. Saha-Dasgupta, O. Jepsen, and O. K. Andersen, Phys. Rev. Lett. 87, 047003 (2001).

${ }^{27}$ T. Valla, A. V. Fedorov, P. D. Johnson, B. O. Wells, S. L. Hulbert, Q. Li, G. D. Gu, and N. Koshizuka, Science 285, 2110 (1999).

${ }^{28}$ D. S. Inosov, J. Fink, A. A. Kordyuk, S. V. Borisenko, V. B. Zabolotnyy, R. Schuster, M. Knupfer, B. Büchner, R. Follath, H. A. Dürr, W. Eberhardt, V. Hinkov, B. Keimer, and H. Berger, Phys. Rev. Lett. 99, 237002 (2007).

${ }^{29}$ M. Abdel-Jawad, M. P. Kennett, L. Balicas, A. Carrington, A. P. Mackenzie, R. H. McKenzie, and N. E. Hussey, Nat. Phys. 2, 821 (2006).

${ }^{30}$ S. Nakamae, K. Behnia, N. Mangkorntong, M. Nohara, H. Takagi, S. J. C. Yates, and N. E. Hussey, Phys. Rev. B 68, 100502(R) (2003).

${ }^{31}$ Y. Kakehashi and P. Fulde, Phys. Rev. Lett. 94, 156401 (2005).

${ }^{32}$ C. M. Varma, Phys. Rev. Lett. 83, 3538 (1999).

${ }^{33}$ Sudip Chakravarty, R. B. Laughlin, Dirk K. Morr, and Chetan Nayak, Phys. Rev. B 63, 094503 (2001).

${ }^{34}$ Eun-Ah Kim, Michael J. Lawler, Paul Oreto, Subir Sachdev, Eduardo Fradkin, and Steven A. Kivelson, Phys. Rev. B 77, 184514 (2008).

${ }^{35}$ Andrea Pelissetto, Subir Sachdev, and Ettore Vicari, Phys. Rev. Lett. 101, 027005 (2008).

${ }^{36}$ P. A. Lee, N. Nagaosa, and X.-G. Wen, Rev. Mod. Phys. 78, 17 (2006).

${ }^{37}$ P. W. Anderson, Nat. Phys. 2, 626 (2006). 